\title{
Evolutionary Ecology of Natural Comammox Nitrospira Populations
}

\author{
Palomo, Alejandro; Dechesne, Arnaud; Cordero, Otto X.; Smets, Barth F.
}

Published in:

mSystems

Link to article, DOI:

10.1128/msystems.01139-21

Publication date:

2022

Document Version

Publisher's PDF, also known as Version of record

Link back to DTU Orbit

Citation $(A P A)$ :

Palomo, A., Dechesne, A., Cordero, O. X., \& Smets, B. F. (2022). Evolutionary Ecology of Natural Comammox Nitrospira Populations. mSystems, 7(1), [e01139-21]. https://doi.org/10.1128/msystems.01139-21

\section{General rights}

Copyright and moral rights for the publications made accessible in the public portal are retained by the authors and/or other copyright owners and it is a condition of accessing publications that users recognise and abide by the legal requirements associated with these rights.

- Users may download and print one copy of any publication from the public portal for the purpose of private study or research.

- You may not further distribute the material or use it for any profit-making activity or commercial gain

- You may freely distribute the URL identifying the publication in the public portal

If you believe that this document breaches copyright please contact us providing details, and we will remove access to the work immediately and investigate your claim. 


\title{
Evolutionary Ecology of Natural Comammox Nitrospira Populations
}

\author{
(D) Alejandro Palomo, ${ }^{\text {a* } \text { (D) Arnaud Dechesne, }}{ }^{a}$ (D) Otto X. Cordero, ${ }^{b}$ (D) Barth F. Smets ${ }^{a}$ \\ aDepartment of Environmental Engineering, Technical University of Denmark, Kongens Lyngby, Denmark \\ bRalph M. Parsons Laboratory for Environmental Science and Engineering, Department of Civil and Environmental Engineering, Massachusetts Institute of Technology, \\ Cambridge, Massachusetts, USA
}

ABSTRACT Microbes commonly exist in diverse and complex communities where species interact, and their genomic repertoires evolve over time. Our understanding of species interaction and evolution has increased during the last decades, but most studies of evolutionary dynamics are based on single species in isolation or in experimental systems composed of few interacting species. Here, we use the microbial ecosystem found in groundwater-fed sand filter as a model to avoid this limitation. In these open systems, diverse microbial communities experience relatively stable conditions, and the coupling between chemical and biological processes is generally well defined. Metagenomic analysis of 12 sand filters communities revealed systematic co-occurrence of at least five comammox Nitrospira species, likely promoted by low ammonium concentrations. These Nitrospira species showed intrapopulation sequence diversity, although possible clonal expansion was detected in a few abundant local comammox populations. Nitrospira species showed low homologous recombination and strong purifying selection, the latter process being especially strong in genes essential in energy metabolism. Positive selection was detected for genes related to resistance to foreign DNA and phages. We found that, compared to other habitats, groundwater-fed sand filters impose strong purifying selection and low recombination on comammox Nitrospira populations. These results suggest that evolutionary processes are more affected by habitat type than by species identity. Together, this study improves our understanding of species interaction and evolution in complex microbial communities and sheds light on the environmental dependency of evolutionary processes.

IMPORTANCE Microbial species interact with each other and their environment (ecological processes) and undergo changes in their genomic repertoire over time (evolutionary processes). How these two classes of processes interact is largely unknown, especially for complex communities, as most studies of microbial evolutionary dynamics consider single species in isolation or a few interacting species in simplified experimental systems. In this study, these limitations are circumvented by examining the microbial communities found in stable and well-described groundwaterfed sand filters. Combining metagenomics and strain-level analyses, we identified the microbial interactions and evolutionary processes affecting comammox Nitrospira, a recently discovered bacterial type capable of performing the whole nitrification process. We found that abundant and co-occurrent Nitrospira populations in groundwater-fed sand filters are characterized by low recombination and strong purifying selection. In addition, by comparing these observations with those obtained from Nitrospira species inhabiting other environments, we revealed that evolutionary processes are more affected by habitat type than by species identity.

KEYWORDS comammox, evolutionary ecology, microdiversity, nitrification, selection
Editor Angela D. Kent, University of Illinois at Urbana-Champaign

Copyright $\odot 2022$ Palomo et al. This is an open-access article distributed under the terms of the Creative Commons Attribution 4.0 International license.

Address correspondence to Barth F. Smets, bfsm@env.dtu.dk.

*Present address: Alejandro Palomo, School of Environmental Science and Engineering, Southern University of Science and Technology, Shenzhen, China.

The authors declare no conflict of interest.

Received 15 September 2021

Accepted 15 December 2021

Published 11 January 2022 
icroorganisms dominate the tree of life based on species diversity, and they play an essential role in all global biogeochemical cycles. Microbial species interact with each other and with the environment (ecological processes) and also undergo changes in their genomic repertoire over time (evolutionary processes). Yet the interaction between ecological and evolutionary processes is largely unknown, especially for complex open communities. For many years, most studies of microbial communities in open, complex environments have focused on ecological aspects, as it was believed that evolutionary changes happen at a much larger timescale (1). However, in recent years, with the development of population genomics analysis, researchers have started to jointly investigate ecological and evolutionary processes. Yet most studies of evolutionary dynamics remain based on single species in isolation (2) or on experimental systems composed of only a few interacting species (3). While these analyses have helped to understand some aspects of evolutionary patterns, they have limitations because they lack many characteristics of true natural populations (e.g., spatial structure, existence of microdiversity, predation, immigration). On the other hand, observing populations in the wild also has limitations because the conditions vary with little control (hence, uncontrolled variation in population size, selection regime) and because the typically unknown ecophysiology of retrieved genomes makes it difficult to interpret the observed patterns. Therefore, studying well-defined model microbial ecosystems can help to understand ecological and evolutionary processes in microbial communities (4).

Rapid sand filters (RSF), widely used to produce drinking water from groundwater, are useful model systems. They are characterized by stable conditions, including active growth, primarily driven by the oxidation of ammonia, methane, and other inorganic compounds present at low concentrations in the influent water, large populations $\left(10^{9}\right.$ to $10^{10}$ cells/g), significant mixing (due to backwashing), continuous but limited immigration from prokaryotes in the influent water, and no dispersal between separate sand filters (resulting in allopatric populations) (5-8). In addition, the microbial communities inhabiting these systems, which are usually stable across time (9), have been broadly described, showing a general dominance of complete ammonia oxidizers (comammox) $(6,10,11)$. These recently discovered microorganisms are expected to have a relatively simple ecology (due to their chemolithoautotrophic metabolism) (12), yet are poorly studied in terms of what drives their diversity, distribution, and evolution. Furthermore, as comammox bacteria occur in RSF as coexisting populations (10, 13), RSF offer an opportunity for resolving fine-scale genomic heterogeneity within closely related strains and investigating if they show similar patterns in evolutionary processes (such as selection or recombination).

Of particular interest is to determine to what extent the evolutionary processes that drive the diversification of comammox Nitrospira are dependent on their environment, as opposed to intrinsic properties of the species. The environmental dependency of microbial evolution has been investigated from different perspectives. Several studies have focused on genome signature variations (GC, tetranucleotide signatures, codon usage, purine-pyrimidine ratio) associated with different environments (reviewed in Dutta and Paul [14]). Others have studied bacterial adaptation to shifting environments (15) or have targeted a specific evolutionary process across several lifestyles (e.g., homologous recombination [16] or selection [17]). Most of these studies, however, considered different species living in different environments or closely related species with a different lifestyle (i.e., free-living organisms versus pathogens). Yet little is known about ongoing evolutionary processes for species belonging to the same lineage with presumed similar physiology inhabiting different open environments. In this study, taking advantage of the multiple comammox species present in several groundwater-fed RSF, we thoroughly investigated evolutionary processes in this environment and compared these observations with those in comammox species inhabiting other environments. 


\section{RESULTS AND DISCUSSION}

We examined ecological and evolutionary patterns within comammox-dominated bacterial communities inhabiting groundwater-fed rapid sand filters. To that end, we retrieved metagenome-assembled genomes (MAGs) from 12 similarly operated waterworks in Denmark, using a combination of automatic and manual binning, followed by several refinement steps to improve the bin quality (Fig. S1 in the supplemental material). To remove redundancy, a single representative was selected for each set of genomes that shared an average nucleotide identity (ANI) greater than $99 \%$. This resulted in a total of 189 MAGs (genome completeness, $83.9 \% \pm 13.6$; contamination, $1.9 \% \pm 1.4$ ) (Table S1 at https://figshare.com/articles/dataset/Table_S1/ 17099123), 18 of them classified as Nitrospira spp. (completeness, 89.1\% \pm 9.2 ; contamination, $2.5 \% \pm 1.1$ ) (Table S2 at https://figshare.com/articles/dataset/Table_S2/ 17099138). These Nitrospira MAGs spanned 16 putative species (further on simply referred to as "species") using a threshold average nucleotide identity (ANI) of $\geq 95 \%$ (18-20). The phylogenomic analysis placed 1 Nitrospira species into lineage I and 14 into lineage II, and another 1 was not assigned to any previously described lineage due to the lack of similar reference genomes (Fig. 1). Of the 16 Nitrospira species, 12 were classified as comammox Nitrospira (5 clade A and 7 clade B) (Fig. 1). As expected, the genomes classified as comammox Nitrospira also contained genes essential for complete ammonia oxidation, such as those of the ammonia monooxygenase (AMO) and hydroxylamine dehydrogenase (HAO) operons. Further description of the Nitrospira MAGs can be found elsewhere (21).

Nitrospira species comprised a large proportion of the microbial communities of the waterworks (27 to 70\%), and comammox represented a large fraction of Nitrospira spp. (76 to 98\%) (Fig. 2 and Table S3 at https://figshare.com/articles/dataset/Table_S3/ 17099141). For most of the RSFs, the variation in the abundance of the different Nitrospira MAGs between duplicate samples was low (Fig. S2), suggesting a high spatial homogeneity in the top layer of the filters, probably due to the frequent mixing caused by backwash, and, hence, supporting the representativeness of our sampling. Multiple Nitrospira species (at least 5; 10 on average) co-occurred in all the waterworks (Fig. 2). However, there was no consistent dominance pattern. In some cases, a single species, but not always the same, dominated (waterworks 4 [WW4] and WW5), while in others, two (WW9 and WW12) or more species had similar high abundance (Fig. 2). The chemical characteristics of the water explained $57 \%$ of the variance in Nitrospira composition (permutation test, $P<0.001$ ) (Table S4 at https://figshare.com/articles/dataset/Table _S4/17099150), suggesting that water chemistry is a strong filter for the assembly of these nitrifying communities. Among the measured water constituents, the influent ammonium concentration best explained Nitrospira distribution (explained 18\%; permutation test, $P=0.02$ ) (Table S4 at https://figshare.com/articles/dataset/Table_S4/ 17099150). Higher comammox species richness was detected in waterworks treating lower ammonium concentration (Fig. S3) $\left(R^{2}=0.54 ; P<0.01\right)$. Canonical Nitrospira and canonical ammonia oxidizers were more abundant in waterworks receiving influent with higher ammonium (Fig. S4). These observations are in line with the prediction that higher ammonium concentration favors the division of labor between canonical ammonia and nitrite oxidizer (22). Nevertheless, we observed that one comammox species (RSF3) seemed to cope with slightly higher ammonium concentrations as well (Fig. S4). Interestingly, besides the mentioned RSF3, the other comammox Nitrospira species tended to cluster with other members of the same clade (clades are separated based on the phylogeny of ammonia monooxygenase subunit) (Fig. S4). The distribution pattern of Nitrospira species across the waterworks was not related to their geographic distance (Mantel test; $r$ statistic, 0.08; significance $>0.05$ ).

Significant correlation between the abundances of the comammox species was rare; only a few strong positive correlations were detected (RSF5 and RSF15_CG24_B [ $\rho=0.84$ ]; RSF6 and RSF11 [ $\rho=0.84]$ ), while no significant negative correlations were observed (Fig. S5). This absence of obvious competitive exclusion pattern, together 


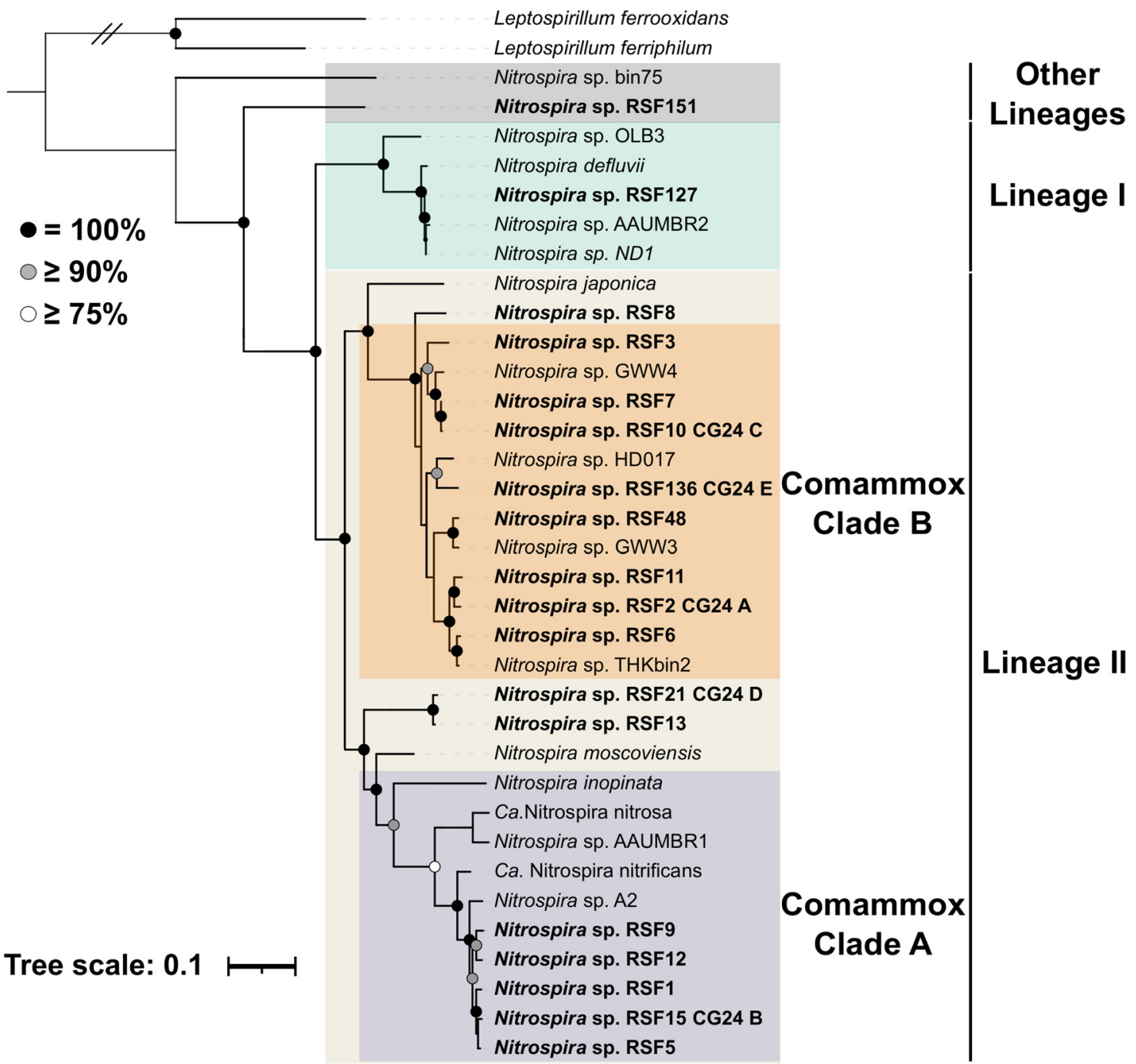

FIG 1 Phylogenomic affiliation of Nitrospira MAGs retrieved from 12 waterworks. A phylogenetic tree was built based on the concatenation of 120 proteins. Nitrospira MAGs retrieved in this study are highlighted in boldface. Lineages and sublineages are shown with colors (lineage I, green; lineage II, light brown; other lineages, gray; comammox clade A purple; comammox clade B, orange). Leptospirillum was used to root the tree. The strength of support for internal nodes as assessed by bootstrap replicates is indicated as colored circles (top left legend).

with the aforementioned description of the coexistence of multiple comammox species, suggests that these microorganisms exploit slightly different niches. An explanation might be that the co-occurrent comammox Nitrospira species had different ammonia affinities, and they would occupy different sites in the highly porous filter material (23). In fact, ammonium concentration was the variable that better explained the Nitrospira distribution in the studied waterworks. Although little is known about the range of ammonia affinities in comammox Nitrospira, as this trait has only been measured in two species $(24,25)$, other ammonia oxidizers have ammonia affinities spanning several orders of magnitude (26). Likewise, canonical Nitrospira species affiliated with different lineages have shown niche differentiation based on nitrite concentration $(27,28)$. Another explanation could be a dissimilar use of alternative metabolisms. It has been documented that some Nitrospira species can use hydrogen as energy source (29) and formate as carbon and/or energy source (depending on the Nitrospira type) (28). While some of the comammox genomes recovered from the waterworks encode genes for formate oxidation, others harbor genes putatively involved in hydrogen metabolism (Table S5 at https://figshare.com/articles/dataset/Table_S5/17099153). 


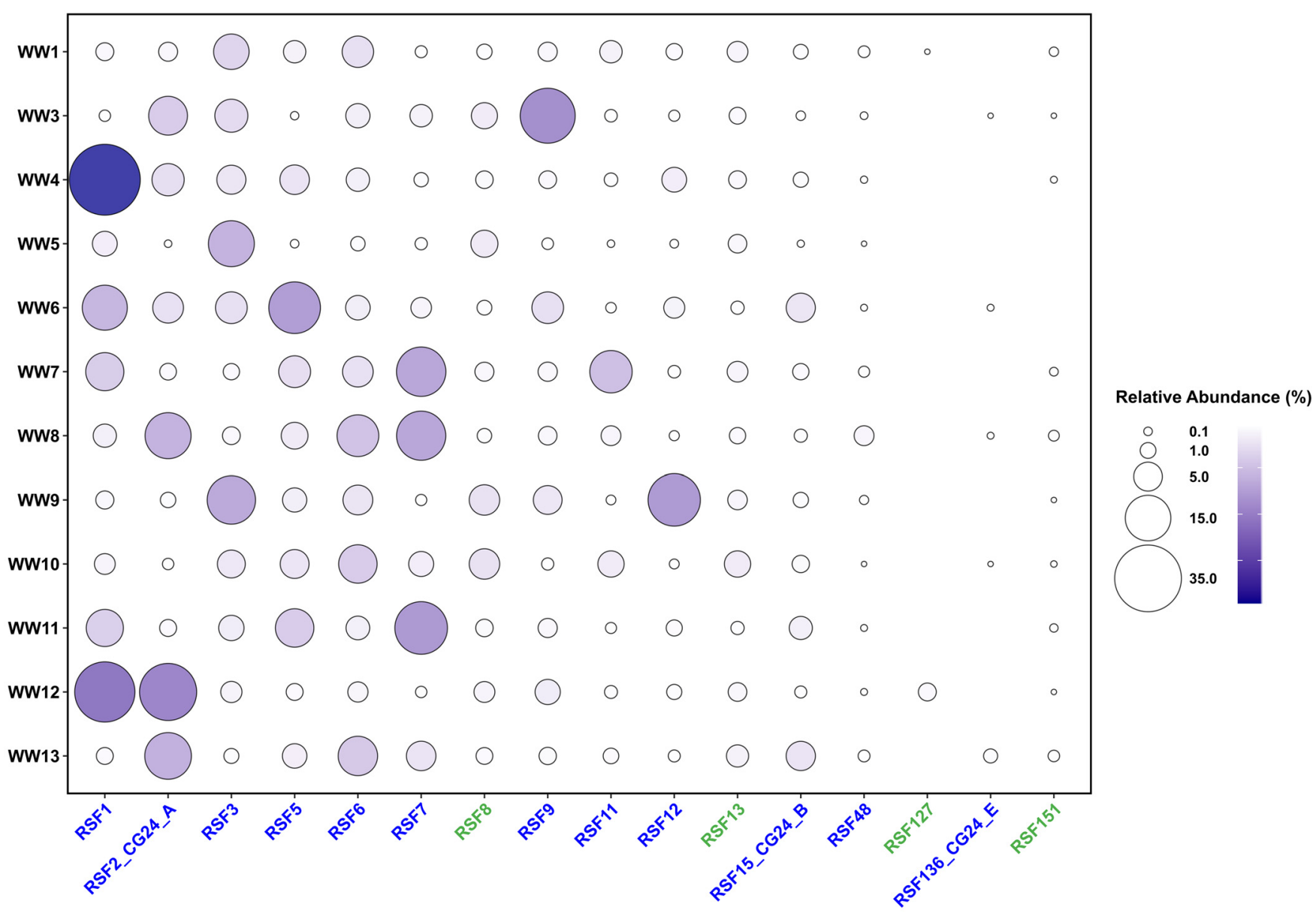

FIG 2 Abundance of Nitrospira species across 12 waterworks. Relative abundance (related to the total community) of 16 Nitrospira species in 12 waterworks. Comammox and canonical Nitrospira species are denoted in blue and green, respectively. Only relative abundances above $0.005 \%$ are considered.

Another feature that may contribute to niche differentiation is the distinct $\mathrm{N}$ assimilation capacity, as the comammox genomes contain transporters with different ammonium affinity and uptake capacity (13) (Table S5 at https://figshare.com/articles/ dataset/Table_S5/17099153). Besides this, each comammox Nitrospira species contain a large number of unique genes clusters ( $>250$ on average) (21). Although the function of most of them is unknown, these unique genes might promote ecological variation. Traits such as chemotactic strategies, attachment to particles strategies, secondary metabolism, or defense against predation have been proposed to explain this phenomenon in other coexisting microorganisms (reviewed in Louca et al. [30]).

Microdiversity within Nitrospira species. Strain-level analysis across the waterworks revealed that the Nitrospira populations contained intraspecies sequence diversity. We exploited the shotgun metagenomic data to perform strain-level analyses on the 12 most abundant Nitrospira species (genome completeness, $92.4 \% \pm 5.5$; contamination, $2.7 \% \pm 0.8$ ) based on single nucleotide polymorphisms (SNPs). The number of SNPs/Mbp in the populations across the waterworks ranged from 14,437 to 45,664 (Table S6 at https://figshare.com/articles/dataset/Table_S6/17099159). Looking into the populations at the local scale (species within waterworks), the number of SNPs/ Mbp ranged from 249 to 37,663 (Table S7 at https://figshare.com/articles/dataset/ Table_S7/17099171).

We observed a wide range of microdiversity (measured as nucleotide diversity $[\pi]$ ) among species (Fig. 3A): canonical Nitrospira RSF8 was the most diverse species, with three times more nucleotide diversity than the less diverse Nitrospira species of our study (RSF1 and RSF12) (Fig. 3A). Depending on the Nitrospira population, we detected 


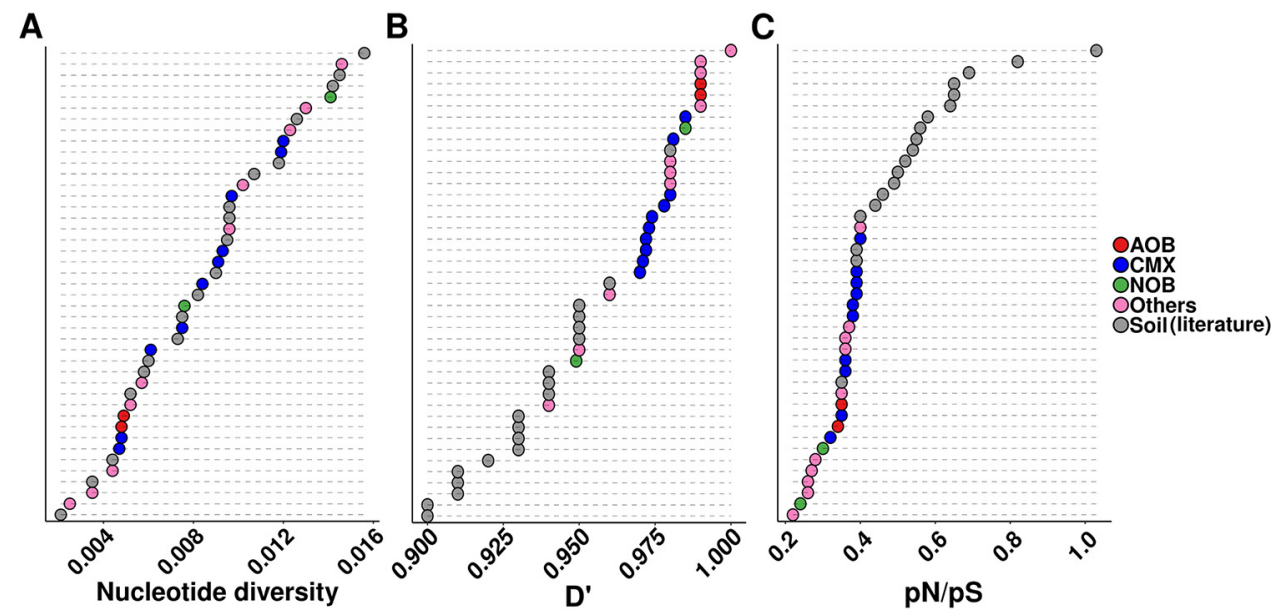

FIG 3 Evolutionary metrics of Nitrospira populations across 12 waterworks compared to other organisms and locations. (A) Nucleotide diversity $(\pi)$ of the most abundant bacterial populations across 12 waterworks and the most abundant bacterial populations across a grassland meadow (denoted as "soil") (38). (B) Homologous recombination $\left(D^{\prime}\right)$ of most abundant bacterial populations across 12 waterworks and most abundant bacterial populations across grassland meadow. (C) Selection (pN/pS ratio) of most abundant bacterial populations across 12 waterworks and most abundant bacterial populations across grassland meadow. AOB, ammonia-oxidizing bacteria; CMX, comammox; NOB, nitrite oxidizing bacteria; others, nonnitrifying abundant bacteria present in the waterworks. The data reported in this figure (including the MAGs ID) can be found at https://figshare.com/articles/dataset/Table_S6/17099159.

instances of both homogeneous microdiversity across the waterworks (e.g., RSF5 and RSF8), as well as a high microdiversity variation depending on the waterworks (e.g., RSF1, RSF9, and RSF11) (Fig. S6). Based on our observations of high species-level comammox Nitrospira diversity at low ammonium concentration, we hypothesized that such conditions also promote high microdiversity. However, this was not the case, as the correlations of microdiversity with ammonium concentration or with comammox species richness were not significant for any species $(P>0.05)$. This suggests different drivers for interspecies versus within-species diversities.

While all species showed significant microdiversity across waterworks, we detected a few highly abundant comammox populations with almost no microdiversity at the local scale (e.g., comammox Nitrospira RSF1 and RSF12 in WW4 and WW9, respectively) (Fig. S7), which suggests local clonal expansions of these comammox populations. Along the same line, the analysis of major allele frequencies of common SNPs revealed that only a fraction of the within-species variants diversity is found locally, and in some cases, distinct single variants dominated different waterworks (e.g., comammox RSF3 in WW10B versus WW5) (Fig. S8). These results contrast with species-level observations, where all the diversity was represented in each waterworks (as they all contain most of the Nitrospira species) (Fig. 2).

Similar to what we observed at the species level, there was no significant correlation between similarity in subspecies composition and the geographic distance of the waterworks, with the exceptions of Nitrospira sp. RSF2 $(P<0.01)$ and Nitrospira sp. RSF8 ( $P<0.05)$ (Fig. S9). However, we observed an interwaterworks organization of the genetic structure at most loci across the studied genomes, indicating that the Nitrospira populations were more similar within than between waterworks. For each gene, we calculated pairwise fixation indexes $\left(F_{\mathrm{ST}}\right)$ to measure differences in allele frequencies between populations of the same species found in two distinct waterworks. The mean gene $F_{S T}$ values were $\geq 0.15$ for all Nitrospira species (Fig. S10), the most extreme case being RSF5 $\left(F_{\mathrm{ST}}>0.4\right)$, which suggests a great genetic differentiation among the populations (Fig. S10). In a few species (RSF2, RSF7, and RSF8), a lower spatial structure $\left(F_{\mathrm{ST}}<0.2\right)$ of most alleles between waterworks was observed (Fig. S10). These observations differ from those on soil bacterial populations across a meadow obtained using the same approach, where most populations had mean gene $F_{\mathrm{ST}}$ values 
$<0.05$ (31). These contrasting results are consistent with a higher physical separation across the set of waterworks than across samples extracted from a single meadow, where dispersal is more likely to occur.

We also investigated local regions of the Nitrospira genomes with significantly higher $F_{S T}$ values, as this is characteristic of local (here, in each waterworks) selective pressures (31). Twelve loci with unusually high $\mathrm{F}_{S T}$ were found in six of the Nitrospira populations (Fig. S11 at https://figshare.com/articles/figure/Fig_S11/17099036 and Table S8 at https://figshare.com/articles/dataset/Table_S8/17102996), one of them containing genes involved in nitrogen assimilation (Nitrospira sp. RSF2) (Table S8 at https://figshare.com/articles/dataset/Table_S8/17102996). However, only two of these loci with unusually high site-specific differentiation of alleles (high $F_{\mathrm{ST}}$ ) also had few recombinant events and low nucleotide diversity (Table S8 at https://figshare.com/articles/ dataset/Table_S8/17102996), which signal recent selective sweeps (31). These results suggest that, contrary to what has been observed in several natural populations (31-34), gene-specific sweeps seem to play a minor role in the evolution of Nitrospira species inhabiting the waterworks. A possible explanation could be that the low recombination rate that characterizes the waterworks Nitrospira populations (Fig. 3B, discussed below) limits the possibility of gene-specific sweeps (35).

Overall, across the 12 waterworks, all species presented significant genomic microdiversity, but this diversity was not always represented locally, with a few occurrences of patterns consistent with clonal expansion. The reason for the difference of withinspecies composition across waterworks is unknown, but the allopatric nature of the communities likely contributes to their persistence.

Evolutionary processes at the whole-genome level. The Nitrospira populations were characterized by a low degree of homologous recombination as indicated by the consistently high value of linkage disequilibrium $\left(D^{\prime}\right)$ of their genomes (Fig. 3B), which measures the nonrandom association of alleles at two loci $\left(D^{\prime}\right.$ is only $<1$ if all possible combinations of a pair of biallelic sites are observed [36]; lower $D^{\prime}$ values indicate higher levels of homologous recombination). A similarly low degree of homologous recombination was observed for other abundant non-Nitrospira populations of the waterworks ( $n=12$; genome completeness, $92.6 \% \pm 5.5$; contamination, $2.1 \% \pm 2.0$ ) (Fig. 3B and Table S6 at https://figshare.com/articles/dataset/Table_S6/17099159). In general, this evolutionary process was lower in the waterworks populations than in populations inhabiting a grassland meadow (Fig. 3B), where a similar analysis was conducted (31). To further examine the relative effect of homologous recombination on the genetic diversification of the populations, we measured the rates at which nucleotides become substituted as a result of recombination versus mutation $(\mathrm{r} / \mathrm{m})$. Most of the Nitrospira populations had a relatively low $\mathrm{r} / \mathrm{m}(\mathrm{r} / \mathrm{m}<2)$ compared to recombinogenic species reported in literature $(r / m>4)$ (37) (Fig. S12 at https://figshare.com/articles/ figure/Fig_S12/17099099), although in one case (RSF15_CG24_B), the rate was similar to the value reported for a Streptomyces flavogriseus population $(r / m=28)$ considered to be approaching panmixia (38). Overall, these results suggest a low effect of recombination in the populations of the waterworks. An increasing recombination rate has been associated with fluctuating environments as a source of variation which can accelerate adaptation favoring survival in this type of environment $(39,40)$. On the other hand, constant environments, such as the waterworks studied here, tend to reduce the recombination rate of their residents (39).

The Nitrospira populations were characterized by strong purifying selection. We used the relation between nonsynonymous and synonymous polymorphisms (pN/pS) to investigate this evolutionary process. We detected $\mathrm{pN} / \mathrm{pS}$ of $<1$, indicating purifying selection, for all Nitrospira species (Fig. 3C). Similar results were observed for other abundant populations of the waterworks (Fig. 3C). Purifying selection has frequently been observed in populations in the environment, and it was the case for populations in a grassland meadow (31) (pN/pS, $0.56 \pm 0.17 ; n=19)$, but this process seems to be especially strong in the waterworks populations (pN/pS, $0.34 \pm 0.05 ; n=24$ ) (two- 


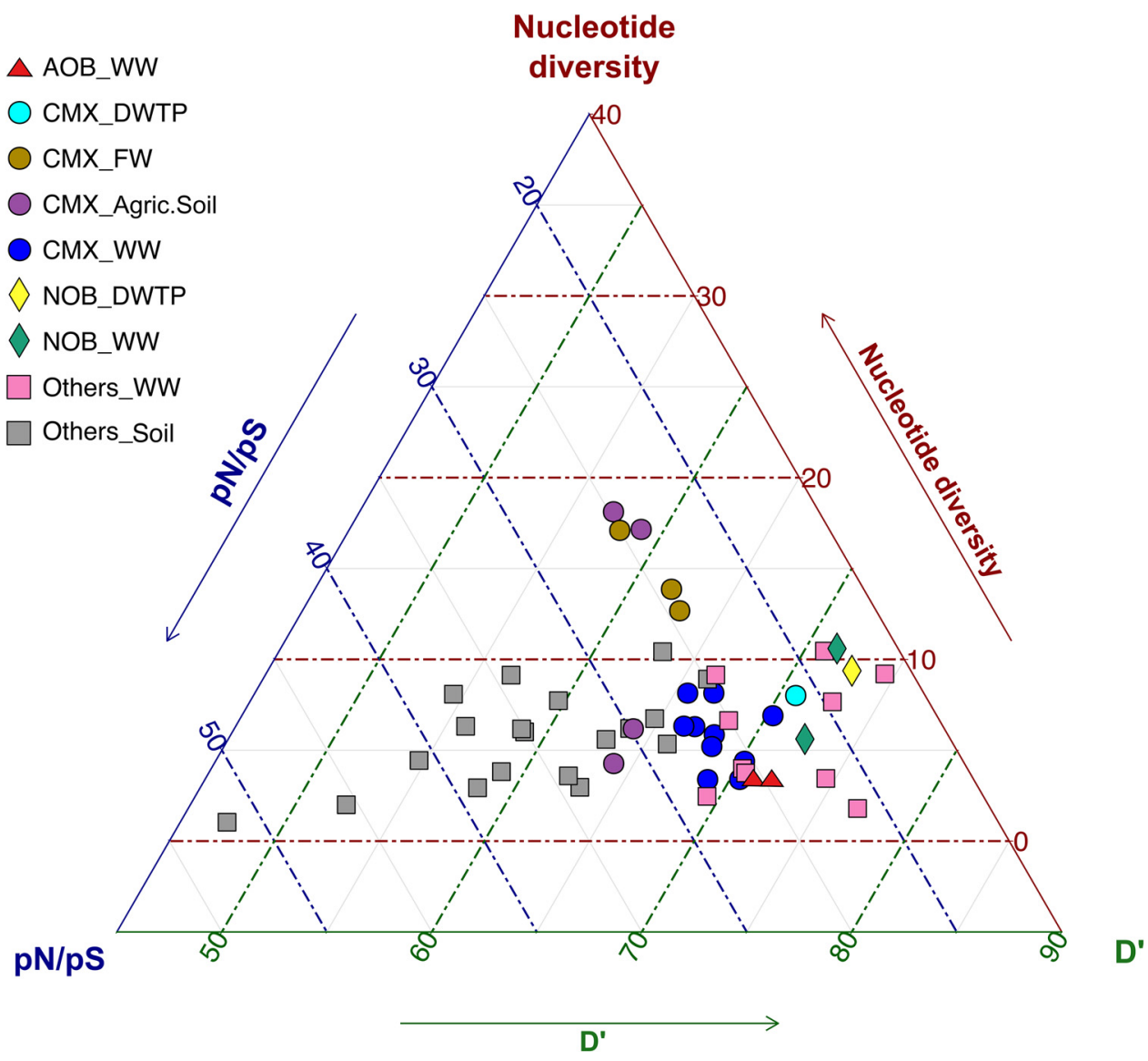

FIG 4 Impact of environment and microbial type in evolutionary metrics. Triplot composed of the nucleotide diversity, $\mathrm{pN} / \mathrm{pS}$ ratio, and $D^{\prime}$ values for the bacterial populations of this study's waterworks (WW), the most abundant bacterial populations across a grassland meadow (38) (Soil) and other abundant Nitrospira populations recovered from other systems. FW, freshwater; DWTP, drinking water treatment plan; Agric.Soil, agricultural soil). The data reported in this table (including the MAG IDs) can be found at https://figshare.com/ articles/dataset/Table_S6/17099159.

sample $t$ test, $P<0.0001$ ) (Fig. 3C). This suggests that their genomes could have reached an adaptive optimum for this stable environment, which is maintained by purging nonsynonymous mutations.

Interestingly, the degree of recombination and diversity across different Nitrospira populations varied substantially with habitat (Fig. 4). High variability of recombination in closely related bacterial species has occasionally been reported (41), and lifestyle appears as one of the most important factors to explain this variability $(16,41)$. Our analysis of Nitrospira populations from different habitats (drinking water treatment plants [DWTP], freshwaters, and soils) suggests that the environment also influences evolutionary processes in free-living bacteria: different bacterial types in the same environment tended to share similar features (Fig. 4), while the evolutionary characteristics of comammox Nitrospira populations differed depending on the environment from where they were retrieved (Fig. 4). Comammox species in the studied waterworks and other DWTP were characterized by low recombination, strong purifying selection, and moderate microdiversity (Fig. 4). In contrast, comammox from freshwater and soils had higher microdiversity and, especially, recombination rate (Fig. 4 and Table S6 at https://figshare.com/articles/dataset/Table_S6/17099159). Intriguingly, we consistently observed that canonical Nitrospira species showed features similar to those of comammox Nitrospira but with even stronger purifying selection (Fig. 4). This feature, together with the much lower richness observed in canonical Nitrospira than comammox bacteria (Fig. 2), suggests that competition can play a more intense role in canonical 

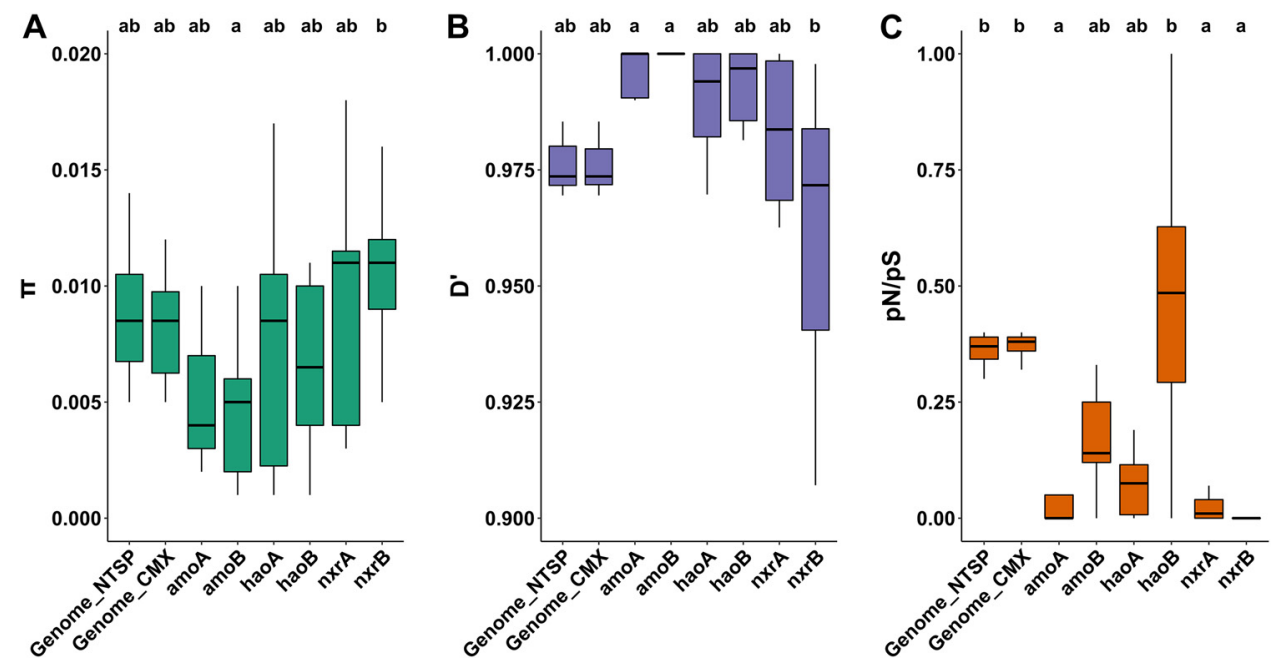

FIG 5 Evolutionary metrics of nitrification genes in Nitrospira populations across 12 waterworks. (A) Boxplot of nucleotide diversity of Nitrospira bacterial populations for whole genome (all Nitrospira and comammox Nitrospira) and nitrification genes. Differences between the mean nucleotide diversities were assessed by a Dunn's test; the same letter means no significant difference $(P>0.05)$. (B) Boxplot of linkage disequilibrium of Nitrospira bacterial populations for whole genome (all Nitrospira and comammox Nitrospira) and nitrification genes. Differences between the mean linkage disequilibriums were assessed by a Dunn's test; the same letter means no significant difference $(P>0.05)$. (C) Boxplot of $\mathrm{pN} / \mathrm{pS}$ ratios of Nitrospira bacterial populations for whole genome (all Nitrospira and comammox Nitrospira) and nitrification genes. Differences between the mean $\mathrm{pN} / \mathrm{pS}$ ratios were assessed by a Dunn's test; the same letter means no significant difference $(P>0.01)$.

Nitrospira, which might select for few species optimally adapted to this type of stable environment. However, a broader analysis is required to confirm this hypothesis.

Evolutionary processes at the gene level. In addition to a genome-wide analysis, we investigated the evolutionary processes at the gene level. In the studied Nitrospira populations, genes involved in nitrification (ammonia monooxygenase, amoA and $a m o B$; hydroxylamine dehydrogenase, $h a \circ A$ and $h a \circ B$; nitrite oxidoreductase, $n x r A$ and $n \times r B$ ) generally had a similar nucleotide diversity (Fig. $5 \mathrm{~A}$ ) and homologous recombination rate $\left(D^{\prime}\right)$ (Fig. 5B) compared to the rest of the genome, but with higher levels of purifying selection (pN/pS) (Fig. 5C). The nucleotide diversities of genes related to nitrification were very similar, with the exception of $a m o B$, which had a significantly lower nucleotide diversity than $n x r B(P<0.05)$ (Fig. 5A). A similar pattern was detected for the recombination, but in this case, $a m o A$, as well as $a m o B$, had significantly lower recombination than $n \times r B(P<0.05)$ (Fig. $5 B)$. We observed a very strong purifying selection for most of the nitrifying genes, especially for $\operatorname{amo} A, n x r A$, and $n x r B(P<0.01)$ (Fig. 5C). In the case of $n \times r B$, not a single nonsynonymous mutation was found in most Nitrospira species ( 0 to 1 nonsynonymous site versus 17 to 66 synonymous sites), even though this gene had a higher nucleotide diversity and homologous recombination (Fig. 5A and B). Our observations on selection are in line with previous studies, as, generally, essential genes and enzymes catalyzing reactions that are difficult to bypass through alternative pathways are subject to higher purifying selection than nonessential ones (42-45).

Even though the average pN/pS values were below 1 in all Nitrospira species (Fig. 3), indicating purifying selection, genes with $\mathrm{pN} / \mathrm{pS}$ values above 1 and significantly higher than the genomic average were detected in each species (Fig. S13 at https://figshare .com/articles/figure/Fig_S13/17099114). Many of those genes under positive selection were related to defense mechanisms against phages (e.g., genes putatively involved in phage entry into cells, ribonucleases, genes coding proteins associated with restrictionmodification systems, and genes related to toxin-antitoxin systems) (Table S9 at https:// figshare.com/articles/dataset/Table_S9/17102999). Comparable findings were made in other abundant species from the waterworks (Table S10 at https://figshare.com/articles/ 
dataset/Table_S10/17103002), in the additional Nitrospira populations retrieved from other environments (Table S10 at https://figshare.com/articles/dataset/Table_S10/17103002), as well as in E. coli and Vibrio sp. strains, respectively $(46,47)$. These observations suggest that positive selection in phage defense-related genes is widespread across bacteria and highlights the evolutionary arms race occurring between phages and bacteria as an important driver in bacterial ecology and evolution $(35,48,49)$. Additionally, we found nondefense mobile genetic elements, such as transposons and integrases, with significantly higher $\mathrm{pN} / \mathrm{pS}$ values than the genome average in the Nitrospira spp. (Table S11 at https://figshare.com/articles/dataset/Table_S11/17103008).

Conclusions. A major unresolved question is how the relationship between ecology and evolution shapes complex communities in the environments. Here, we used a model microbial system with relatively stable conditions to examine this question. By analyzing the microbial communities in 12 groundwater-fed rapid sand filters, we observed that co-occurring comammox Nitrospira spp. dominate all sites, but with local differences in species composition. This suggests that the abundant comammox Nitrospira spp. exploit slightly different niches, which could partially be explained by the inlet water chemistry composition and their different genetic repertoires. At the subspecies level, comammox Nitrospira spp. are characterized by strong purifying selection and low recombination. These features, together with the occasional genome sweeps we detected, suggest that the subspecies possibly occupy a narrow niche to which they are optimally adapted. In contrast, we also showed that the relative magnitude of these evolutionary processes was different in comammox Nitrospira in habitats where environmental conditions are less stable and immigration more intense. Thus, we conclude that the evolutionary processes that drive the diversification of Nitrospira are dependent on the environment, as opposed to intrinsic properties of the species.

\section{MATERIALS AND METHODS}

Sampling, sequencing, and metagenomic assembled genomes recovery. The sampling description, DNA extraction, and sequencing have been previously described (21). Briefly, filter material was collected from two locations at the top of the filters of 12 Danish waterworks. DNA was extracted from $0.5 \mathrm{~g}$ of sand material using the MP FastDNA Spin kit (MP Biomedicals LLC, Solon, OH, USA). DNA libraries were generated using the 24 extracted DNA with the Nextera XT DNA library preparation kit (Illumina Inc.) according to the manufacturer's instructions. Samples were sequenced in one lane, with $2 \times 150$ paired-read sequencing on the Illumina HiSeq4000 at BGl's facility in Copenhagen. Generated reads were trimmed and filtered, including adapters removal, using Trimmomatic v.0.22 (threshold quality, 15; minimum length, 40) (50). FastQC (Babraham Bioinformatics [http://www.bioinformatics.babraham.ac.uk/ projects/fastqc/]) was used to evaluate the quality of the obtained reads. Coassembly of high-quality reads was conducted using IDBA-UD (51) with the options -pre_correction -min_contig 1000. Additionally, single-sample assemblies were performed following the same procedure. As it has been shown that the combination of multiple binning algorithms outperforms the usage of one single algorithm (52), here, we performed metagenomic binning using MetaBAT (53), MaxBin2 (54), CONCOCT (55), MyCC (56), BinSanity (57), and COCACOLA (58) (Fig. S1 in the supplemental material). The quality of the resulting genomes was improved using Binning_refiner (59). Simultaneously, we ran the software metaWRAP (60), which also take advantage of multiple binning tools to recover genomes from the coassembly. The generated bins were improved using the refinement module of metaWRAP. Selection of the best genomes recovered with the different binning algorithms was done with DAS Tool (61). dRep (62) was applied to dereplicate all the selected best bins with the secondary clustering threshold set at $99 \%$ genome-wide average nucleotide identity (gANI). On the other hand, mmgenome (63) was applied to the single-sample assemblies to recover Nitrospira genomes following the strategy described elsewhere (13). The generated Nitrospira genomes were combined with the dereplicated bins and subjected to the reassembly module of metaWRAP with the aim of improving the quality of the bins. dRep at $99 \%$ gANI was used again for dereplication. RefineM (-cov_corr $=0.8)(64)$ was used to refine the resulting dereplicated bins by removing scaffolds with divergent GC content or tetranucleotide frequencies. Furthermore, the binning and refinement modules from metaWRAP were applied to the coassembly of the six RSF samples used in Palomo et al. (5). Obtained bins, together with the reported ones in Palomo et al. $(5,13)$, were dereplicated using dRep at $99 \%$ gANI. A refinement step with RefineM was applied on the resulting dereplicated bins. These refined bins, together with the bins recovered from the 12 waterworks of this study, were dereplicated as described above. In addition, the assembly quality of the Nitrospira bins was improved by alignment against related complete or draft genomes using the multidraft-based scaffolder (MeDuSa) (65). The overall procedure here described can be visualized in Fig. S1. The completeness and contamination of the bins were evaluated using CheckM (66).

Species abundance estimation. A 95\% average nucleotide identity (ANI) cutoff was used to define species as proposed by Klappenbach et al. (18). The retrieved MAGs were dereplicated using dRep with 
the secondary clustering threshold set at $95 \%$ gANI. Among the genomes classified as belonging to the same species, the one with higher quality was chosen as representative genome. The species' abundance and coverage of each representative genome across the metagenomes were assessed using MIDAS (67). Briefly, MIDAS uses reads mapped to 15 universal single-copy gene families (with ability to accurately recruit metagenomic reads to the correct species [67]) to estimate the abundance and coverage of bacterial species from a shotgun metagenome. We used the species retrieved in this study to build the database of universal single-copy genes.

Genome classification and annotation. MAGs were classified using the classify workflow of the GTDB-Tk v.0.1.3 tool (68). Open reading frames were predicted using Prodigal v.2.63 (69) and annotated using BLASTP (70) against NCBI nr (71), UniProt (72), KEGG (73), PFAM (74), and eggNOG (75). Genes were assigned to antiphage defense systems using the strategy described in Doron et al. (76).

Phylogenetic analysis. Phylogenetic analyses of Nitrospira genomes were conducted with the GTDB-Tk v.0.1.3 tool $(68,21)$ using the de novo workflow with a set of 120 single-copy marker proteins and the genome taxonomy database (GTDB) (77). Concatenated alignments were used to construct a maximum-likelihood tree using RAXML v.8.2.11 (78) with 200 rapid bootstraps (determined using the autoMRE option) and the LG likelihood model of amino acid substitution with gamma-distributed rates and fraction of invariant sites (-m PROTGAMMAILGF; best model determined using ProtTest v.3.4.2 [79]). The tree was rooted using two Leptospirillum species as outgroup. The rooted tree was visualized using the online web tool from the Interactive Tree of Life (iTol) (80).

Read mapping, SNP calling, and population genomic analysis. The population genomic analysis was done following the approach described in Crits-Christoph et al. (31). High-quality reads were mapped to an indexed database of the 176 species MAGs recovered from the waterworks using BWA-MEM (81). The resulting alignments were filtered using SAMtools (82) view -q30 to remove reads with mapping quality less than 30, and also with the script filter_reads.py (31) (with the options - $m$ 96 to retain reads with a percent identity of at least $96 \%$ to the reference and -q 2 to ensure uniquely best mapping read pairs in the index). Downstream population genomic analysis was performed on the 24 most abundant species genomes (12 Nitrospira ones and 12 other abundant species genomes). For each of these species' genomes, we analyzed data in samples that passed a cutoff of at least $50 \%$ of the genome being covered with at least $5 \times$ coverage. One hundred forty-nine out of 576 sample genome comparisons ( 24 genomes $\times 24$ samples) passed this minimum requirement. Sample read mappings were pooled by each waterworks and by all samples across the waterworks. Nucleotide diversity $(\pi)$, linkage disequilibrium $\left(D^{\prime}\right)$, and the ratio of nonsynonymous to synonymous polymorphism ( $\mathrm{pN} / \mathrm{pS}$ ) were calculated for each sample, each waterwork, and across all the waterworks as described elsewhere (31) using the scripts provided by the authors. Fixation index $\left(F_{\mathrm{ST}}\right)$, which measures the degree of genetic differentiation, was calculated following the same procedure but on sites segregating across two waterworks being compared (for all the possible waterworks comparisons). As Crits-Christoph et al. (31) recommended, only sites with a coverage of at least $20 \times$ in each waterworks were used to calculate $F_{\mathrm{ST}}$. In addition, genes with coverage in a waterworks outside the range of two standard deviations were excluded from the analysis. As previously suggested (31), a two-sample Wilcoxon test was conducted to find out if average linkage of highly differentiated loci differed from the genomic average for each species. Similarly, a two-sample $t$ test was used to conclude if average nucleotide diversity of highly differentiated loci differed from the genomic average. Both sets of tests were corrected for multiple hypotheses using the Benjamini-Hochberg method. Recombination to mutation ratio was inferred using mcorr (37).

The strain-level analysis described above was also conducted in other Nitrospira MAGs previously recovered (21). Only those ones that passed a cutoff of at least $50 \%$ of the genome being covered with at least $5 \times$ coverage in any of the metagenomes where the MAGs were present (21) were kept for further analysis.

Statistical analyses. All statistical tests were performed using R v.3.5.2 (83). Due to the compositional nature of sequencing data (84), for all statistical analyses, species abundances were analyzed as follows: zeros were replaced with an estimate value using the count zero multiplicative approach with the $\mathrm{ZCompositions} \mathrm{R} \mathrm{package} \mathrm{(85),} \mathrm{and} \mathrm{data} \mathrm{were} \mathrm{further} \mathrm{centered} \mathrm{log-ratio} \mathrm{transformed.} \mathrm{Nitrospira} \mathrm{com-}$ munity dissimilarities were calculated using the Jaccard index. The correlation between the Nitrospira community dissimilarities and geographic distances was calculated using the Mantel test (significance obtained after 100,000 permutations). The same analysis was used to assess the correlation between the Nitrospira community dissimilarities and the water composition dissimilarity, as well as the correlation between major allele dissimilarities and geographic distances.

Proportionality between abundances of the species across the 24 metagenomes were calculated using the propr R package (86) (with the options metric "rho" "ivar $=c \mid r$ ") and visualized using the corrplot $\mathrm{R}$ package (87). For the network analysis, the function getNetwork from propr $\mathrm{R}$ package was used to retain proportionalities $>0.56$ (false-discovery rate $[F D R]<5 \%$ ). The network was visualized using the igraph R package (88).

Redundancy analysis (RDA) was performed in a stepwise way using the ordiR2step function of the vegan package (89). The analysis was conducted using centered log-ratio-transformed Nitrospira species abundances and chemical data of influent water. The constrained ordination model and the variable significance were determined by permutation tests (1,000 permutations) with anova.cca in vegan. Before this analysis, collinearity among explanatory variables was evaluated using variance inflation factor (VIF) in the fmsb package (90). The function vif_func was used to perform a stepwise approach until all variables above a VIF of 5 were excluded (91). Ternary plot was performed using the R package ggtern (92) using the nucleotide diversity, $\mathrm{pN} / \mathrm{pS}$ ratio, and $D^{\prime}$ values for the bacterial populations retrieved from the waterworks, as well as most abundant bacterial populations across grassland meadow (31) and other 
Nitrospira populations abundant in other systems (Table S6 at https://figshare.com/articles/dataset/ Table_S6/17099159).

Differences between the mean nucleotide diversities of the nitrifying genes, whole Nitrospira genomes, and whole comammox Nitrospira genomes were assessed using Kruskal-Wallis analysis of variance (ANOVA) followed by Dunn's test with the Holm-Bonferroni correction. The same analysis was performed for linkage disequilibrium and $\mathrm{pN} / \mathrm{pS}$ ratios.

Chemical analysis of influent water. Ammonium was measured using a standard colorimetric salicylate and hypochlorite method (93), while nitrite was analyzed using a standard method adapted from Grasshoff et al. (94). Nitrate and sulfate were measured by ion chromatography according to American Water Works Association-Water Environment Federation (AWWA-WEF) method 4110 (95). Iron, manganese, and copper were determined by inductively coupled plasma mass spectrometry (ICP-MS) $(7700 \times$; Agilent Technologies), while calcium was determined by ICP-optical emission spectrometry (ICP-OES) (Varian, Vista-MPX charge-coupled device [CCD] Simultaneous ICP-OES). Dissolved oxygen and pH were measured with a handheld meter (WTW Multi 3430, with FDO 925 and SenTix 940 probes).

Data availability. All raw sequence data and Nitrospira genomes retrieved from the Danish rapid sand filters have been deposited at the NCBI BioProject database under accession number PRJNA384587. The rest of the retrieved draft genomes from the Danish rapid sand filters are available on figshare (https://figshare.com/articles/dataset/MAGs_recovered_from_Rapid_Sand_Filters/12962075). Data produced from the strain-level analysis (nucleotide diversity, SPNs, linkage statistics and $F_{\mathrm{ST}}$ metrics) are available on figshare (https://figshare.com/projects/Evolutionary_ecology_of_natural_comammox _Nitrospira_populations/91217).

\section{SUPPLEMENTAL MATERIAL}

Supplemental material is available online only.

FIG S1, PDF file, $0.2 \mathrm{MB}$.

FIG S2, PDF file, $0.4 \mathrm{MB}$.

FIG S3, PDF file, 0.1 MB.

FIG S4, PDF file, $0.5 \mathrm{MB}$.

FIG S5, PDF file, 0.6 MB.

FIG S6, PDF file, $0.2 \mathrm{MB}$.

FIG S7, PDF file, $0.2 \mathrm{MB}$.

FIG S8, PDF file, 1.2 MB.

FIG S9, PDF file, 0.7 MB.

FIG S10, PDF file, 0.1 MB.

\section{ACKNOWLEDGMENTS}

We thank Gabriel E. Leventhal for helpful discussions on data analysis and theory. This research was supported by a research grant (13391, Expa-N) from Villum Fonden.

A.P. conceived the study and performed the bioinformatic analyses. A.P. and A.D. led interpretation of the results supported by O.X.C. and B.F.S. A.P. drafted the manuscript with input from A.D., O.X.C., and B.F.S. All authors contributed to manuscript revision and approved the final version of the manuscript.

We declare that we have no conflict of interest.

\section{REFERENCES}

1. Hairston NG, Ellner SP, Geber MA, Yoshida T, Fox JA. 2005. Rapid evolution and the convergence of ecological and evolutionary time. Ecol Lett 8: 1114-1127. https://doi.org/10.1111/j.1461-0248.2005.00812.x.

2. Barroso-Batista J, Sousa A, Lourenço $M$, Bergman $M-L$, Sobral D, Demengeot J, Xavier KB, Gordo I. 2014. The first steps of adaptation of Escherichia coli to the gut are dominated by soft sweeps. PLoS Genet 10: e1004182. https://doi.org/10.1371/journal.pgen.1004182.

3. Lawrence D, Fiegna F, Behrends V, Bundy JG, Phillimore AB, Bell T, Barraclough TG. 2012. Species interactions alter evolutionary responses to a novel environment. PLoS Biol 10:e1001330. https://doi.org/10.1371/ journal.pbio.1001330.

4. Denef VJ, Mueller RS, Banfield JF. 2010. AMD biofilms: using model communities to study microbial evolution and ecological complexity in nature. ISME J 4:599-610. https://doi.org/10.1038/ismej.2009.158.

5. Palomo A, Fowler SJ, Gülay A, Rasmussen S, Sicheritz-Ponten T, Smets BF. 2016. Metagenomic analysis of rapid gravity sand filter microbial communities suggests novel physiology of Nitrospira spp. ISME J 10: 2569-2581. https://doi.org/10.1038/ismej.2016.63.
6. Tatari K, Musovic S, Gülay A, Dechesne A, Albrechtsen H-J, Smets BF. 2017. Density and distribution of nitrifying guilds in rapid sand filters for drinking water production: dominance of Nitrospira spp. Water Res 127: 239-248. https://doi.org/10.1016/j.watres.2017.10.023.

7. Gülay A, Fowler SJ, Tatari K, Thamdrup B, Albrechtsen H-J, Al-Soud WA, Sørensen SJ, Smets BF. 2019. DNA- and RNA-SIP rReveal Nitrospira spp. as key drivers of nitrification in groundwater-fed biofilters. mBio 10:e0187019. https://doi.org/10.1128/mBio.01870-19.

8. Hu W, Liang J, Ju F, Wang Q, Liu R, Bai Y, Liu H, Qu J. 2020. Metagenomics unravels differential microbiome composition and metabolic potential in rapid sand filters purifying surface water versus groundwater. Environ Sci Technol 54:5197-5206. https://doi.org/10.1021/acs.est.9b07143.

9. Wagner FB, Diwan V, Dechesne A, Fowler SJ, Smets BF, Albrechtsen H-J. 2019. Copper-induced stimulation of nitrification in biological rapid sand filters for drinking water production by proliferation of Nitrosomonas spp. Environ Sci Technol 53:12433-12441. https://doi.org/10.1021/acs.est.9b03885.

10. Fowler SJ, Palomo A, Dechesne A, Mines PD, Smets BF. 2018. Comammox Nitrospira are abundant ammonia oxidizers in diverse groundwater-fed 
rapid sand filter communities. Environ Microbiol 20:1002-1015. https:// doi.org/10.1111/1462-2920.14033.

11. Poghosyan L, Koch H, Frank J, van Kessel MAHJ, Cremers G, van Alen T, Jetten MSM, Op den Camp HJM, Lücker S. 2020. Metagenomic profiling of ammonia- and methane-oxidizing microorganisms in two sequential rapid sand filters. Water Res 185:116288. https://doi.org/10.1016/j.watres .2020 .116288$.

12. Koch H, van Kessel MAHJ, Lücker S. 2019. Complete nitrification: insights into the ecophysiology of comammox Nitrospira. Appl Microbiol Biotechnol 103:177-113. https://doi.org/10.1007/s00253-018-9486-3.

13. Palomo A, Pedersen AG, Fowler SJ, Dechesne A, Sicheritz-Pontén T, Smets BF. 2018. Comparative genomics sheds light on niche differentiation and the evolutionary history of comammox Nitrospira. ISME J 12:1779-1793. https://doi.org/10.1038/s41396-018-0083-3.

14. Dutta C, Paul S. 2012. Microbial lifestyle and genome signatures. Curr Genomics 13:153-162. https://doi.org/10.2174/138920212799860698.

15. Scheuerl T, Hopkins M, Nowell RW, Rivett DW, Barraclough TG, Bell T. 2020. Bacterial adaptation is constrained in complex communities. Nat Commun 11:754. https://doi.org/10.1038/s41467-020-14570-z.

16. González-Torres P, Rodríguez-Mateos F, Antón J, Gabaldón T. 2019. Impact of homologous recombination on the evolution of prokaryotic core genomes. mBio 10:e02494-18. https://doi.org/10.1128/mBio.02494-18.

17. Martinez JL. 2009. The role of natural environments in the evolution of resistance traits in pathogenic bacteria. Proc Biol Sci 276:2521-2530. https://doi.org/10.1098/rspb.2009.0320.

18. Klappenbach JA, Goris J, Vandamme P, Coenye T, Konstantinidis KT, Tiedje JM. 2007. DNA-DNA hybridization values and their relationship to whole-genome sequence similarities. Int J Syst Evol Microbiol 57:81-91. https://doi.org/10.1099/ijs.0.64483-0.

19. Jain C, Rodriguez-R LM, Phillippy AM, Konstantinidis KT, Aluru S. 2018. High throughput ANI analysis of $90 \mathrm{~K}$ prokaryotic genomes reveals clear species boundaries. Nat Commun 9:5114. https://doi.org/10.1038/s41467 -018-07641-9.

20. Olm MR, Crits-Christoph A, Diamond S, Lavy A, Matheus Carnevali PB, Banfield JF. 2020. Consistent metagenome-derived metrics verify and delineate bacterial species boundaries. mSystems 5:e00731-19. https:// doi.org/10.1128/mSystems.00731-19.

21. Palomo A, Dechesne A, Smets BF. 2019. Genomic profiling of Nitrospira species reveals ecological success of comammox Nitrospira. bioRxiv https://doi.org/10.1101/612226.

22. Costa E, Pérez J, Kreft J-U. 2006. Why is metabolic labour divided in nitrification? Trends Microbiol 14:213-219. https://doi.org/10.1016/j.tim.2006.03.006.

23. Gülay A, Tatari K, Musovic S, Mateiu RV, Albrechtsen HJ, Smets BF. 2014. Internal porosity of mineral coating supports microbial activity in rapid sand filters for groundwater treatment. Appl Environ Microbiol 80: 7010-7020. https://doi.org/10.1128/AEM.01959-14.

24. Daims $H$, Lebedeva EV, Pjevac $P$, Han $P$, Herbold C, Albertsen $M$, Jehmlich $N$, Palatinszky M, Vierheilig J, Bulaev A, Kirkegaard RH, von Bergen M, Rattei T, Bendinger B, Nielsen PH, Wagner M. 2015. Complete nitrification by Nitrospira bacteria. Nature 528:504-509. https://doi.org/10.1038/nature16461.

25. Sakoula D, Koch H, Frank J, Jetten MSM, van Kessel MAHJ, Lücker S. 2021. Enrichment and physiological characterization of a novel comammox Nitrospira indicates ammonium inhibition of complete nitrification. ISME J 15:1010-1024. https://doi.org/10.1038/s41396-020-00827-4.

26. Jung $M-Y$, Sedlacek CJ, Kits KD, Mueller AJ, Rhee S-K, Hink L, Nicol GW, Bayer B, Lehtovirta-Morley L, Wright C, de la Torre JR, Herbold CW, Pjevac P, Daims H, Wagner M. 2021. Ammonia-oxidizing archaea possess a wide range of cellular ammonia affinities. ISME J https://doi.org/10.1038/ s41396-021-01064-z.

27. Maixner F, Noguera DR, Anneser B, Stoecker K, Wegl G, Wagner M, Daims H. 2006. Nitrite concentration influences the population structure of Nitrospira-like bacteria. Environ Microbiol 8:1487-1495. https://doi.org/ 10.1111/j.1462-2920.2006.01033.x.

28. Gruber-Dorninger C, Pester M, Kitzinger K, Savio DF, Loy A, Rattei T, Wagner M, Daims H. 2015. Functionally relevant diversity of closely related Nitrospira in activated sludge. ISME J 9:643-655. https://doi.org/10.1038/ismej .2014.156.

29. Koch H, Galushko A, Albertsen M, Schintlmeister A, Gruber-Dorninger C, Lucker S, Pelletier E, Le Paslier D, Spieck E, Richter A, Nielsen PH, Wagner M, Daims H. 2014. Growth of nitrite-oxidizing bacteria by aerobic hydrogen oxidation. Science 345:1052-1054. https://doi.org/10.1126/science.1256985.

30. Louca S, Polz MF, Mazel F, Albright MBN, Huber JA, O'Connor MI, Ackermann M, Hahn AS, Srivastava DS, Crowe SA, Doebeli M, Parfrey LW.
2018. Function and functional redundancy in microbial systems. Nat Ecol Evol 2:936-943. https://doi.org/10.1038/s41559-018-0519-1.

31. Crits-Christoph A, Olm MR, Diamond S, Bouma-Gregson K, Banfield JF. 2020. Soil bacterial populations are shaped by recombination and genespecific selection across a grassland meadow. ISME J 14:1-25. https://doi .org/10.1038/s41396-020-0655-x.

32. Shapiro BJ, Friedman J, Cordero OX, Preheim SP, Timberlake SC, Szabó G, Polz MF, Alm EJ. 2012. Population genomics of early events in the ecological differentiation of bacteria. Science 336:48-51. https://doi.org/10 $.1126 /$ science.1218198.

33. Rosen MJ, Davison M, Bhaya D, Fisher DS. 2015. Fine-scale diversity and extensive recombination in a quasisexual bacterial population occupying a broad niche. Science 348:1019-1023. https://doi.org/10.1126/science .aaa4456.

34. Bendall ML, Stevens SLR, Chan L, Malfatti S, Schwientek P, Tremblay J, Schackwitz W, Martin J, Pati A, Bushnell B, Froula J, Kang D, Tringe SG, Bertilsson S, Moran MA, Shade A, Newton RJ, Mcmahon KD, Malmstrom RR. 2016. Genome-wide selective sweeps and gene-specific sweeps in natural bacterial populations. ISME J 10:1589-1601. https://doi.org/10.1038/ismej .2015.241.

35. Shapiro BJ, Leducq J-B, Mallet J. 2016. What is speciation? PLoS Genet 12: e1005860. https://doi.org/10.1371/journal.pgen.1005860.

36. VanLiere JM, Rosenberg NA. 2008. Mathematical properties of the measure of linkage disequilibrium. Theor Popul Biol 74:130-137. https://doi .org/10.1016/j.tpb.2008.05.006.

37. Lin M, Kussell E. 2019. Inferring bacterial recombination rates from largescale sequencing datasets. Nat Methods 16:199-204. https://doi.org/10 .1038/s41592-018-0293-7.

38. Doroghazi JR, Buckley DH. 2010. Widespread homologous recombination within and between Streptomyces species. ISME J 4:1136-1143. https:// doi.org/10.1038/ismej.2010.45.

39. Carja O, Liberman U, Feldman MW. 2014. Evolution in changing environments: modifiers of mutation, recombination, and migration. Proc Natl Acad Sci U S A 111:17935-17940. https://doi.org/10.1073/pnas.1417664111.

40. Hanage WP. 2016. Not so simple after all: bacteria, their population genetics, and recombination. Cold Spring Harb Perspect Biol 8:a018069. https://doi.org/10.1101/cshperspect.a018069.

41. Didelot X, Maiden MCJ. 2010. Impact of recombination on bacterial evolution. Trends Microbiol 18:315-322. https://doi.org/10.1016/j.tim .2010.04.002.

42. Luo H, Gao F, Lin Y. 2015. Evolutionary conservation analysis between the essential and nonessential genes in bacterial genomes. Sci Rep 5:13210. https://doi.org/10.1038/srep13210.

43. Dilucca M, Cimini G, Giansanti A. 2018. Essentiality, conservation, evolutionary pressure and codon bias in bacterial genomes. Gene 663: 178-188. https://doi.org/10.1016/j.gene.2018.04.017.

44. Aguilar-Rodríguez J, Wagner A. 2018. Metabolic determinants of enzyme evolution in a genome-scale bacterial metabolic network. Genome Biol Evol 10:3076-3088. https://doi.org/10.1093/gbe/evy234.

45. Zhong C, Han M, Yu S, Yang P, Li H, Ning K. 2018. Pan-genome analyses of 24 Shewanella strains re-emphasize the diversification of their functions yet evolutionary dynamics of metal-reducing pathway. Biotechnol Biofuels 11:193. https://doi.org/10.1186/s13068-018-1201-1.

46. Petersen L, Bollback JP, Dimmic M, Hubisz M, Nielsen R. 2007. Genes under positive selection in Escherichia coli. Genome Res 17:1336-1343. https://doi.org/10.1101/gr.6254707.

47. Rabby A, Chakraborty S, Rahman A, Shakila Rahman S, Soad S, Fatima CK, Chakravorty R. 2015. Identification of the positively selected genes governing host-pathogen arm race in Vibrio sp. through comparative genomics approach. Biojournal Sci Technol 2.

48. Rodriguez-Valera F, Martin-Cuadrado A-B, Rodriguez-Brito B, Pašić L, Thingstad TF, Rohwer F, Mira A. 2009. Explaining microbial population genomics through phage predation. Nat Rev Microbiol 7:828-836. https:// doi.org/10.1038/nrmicro2235.

49. Cordero OX, Polz MF. 2014. Explaining microbial genomic diversity in light of evolutionary ecology. Nat Rev Microbiol 12:263-273. https://doi .org/10.1038/nrmicro3218.

50. Bolger AM, Lohse M, Usadel B. 2014. Trimmomatic: a flexible trimmer for Illumina sequence data. Bioinformatics 30:2114-2120. https://doi.org/10 .1093/bioinformatics/btu170.

51. Peng Y, Leung HCM, Yiu SM, Chin FYL. 2012. IDBA-UD: a de novo assembler for single-cell and metagenomic sequencing data with highly uneven depth. Bioinformatics 28:1420-1428. https://doi.org/10.1093/ bioinformatics/bts174. 
52. Probst AJ, Castelle CJ, Singh A, Brown CT, Anantharaman $K$, Sharon I, Hug LA, Burstein D, Emerson JB, Thomas BC, Banfield JF. 2017. Genomic resolution of a cold subsurface aquifer community provides metabolic insights for novel microbes adapted to high CO2 concentrations. Environ Microbiol 19:459-474. https://doi.org/10.1111/1462-2920.13362.

53. Kang DD, Froula J, Egan R, Wang Z. 2015. MetaBAT, an efficient tool for accurately reconstructing single genomes from complex microbial communities. PeerJ 3:e1165. https://doi.org/10.7717/peerj.1165.

54. Wu Y-W, Simmons BA, Singer SW. 2016. MaxBin 2.0: an automated binning algorithm to recover genomes from multiple metagenomic datasets. Bioinformatics 32:605-607. https://doi.org/10.1093/bioinformatics/btv638.

55. Alneberg J, Bjarnason BS, de Bruijn I, Schirmer M, Quick J, ljaz UZ, Lahti L, Loman NJ, Andersson AF, Quince C. 2014. Binning metagenomic contigs by coverage and composition. Nat Methods 11:1144-1146. https://doi .org/10.1038/nmeth.3103.

56. Lin H-H, Liao Y-C. 2016. Accurate binning of metagenomic contigs via automated clustering sequences using information of genomic signatures and marker genes. Sci Rep 6:24175. https://doi.org/10.1038/srep24175.

57. Graham ED, Heidelberg JF, Tully BJ. 2017. BinSanity: unsupervised clustering of environmental microbial assemblies using coverage and affinity propagation. PeerJ 5:e3035. https://doi.org/10.7717/peerj.3035.

58. Lu YY, Chen T, Fuhrman JA, Sun F. 2016. COCACOLA: binning metagenomic contigs using sequence COmposition, read CoverAge, CO-alignment and paired-end read LinkAge. Bioinformatics 33:791-798. https:// doi.org/10.1093/bioinformatics/btw290.

59. Song WZ, Thomas T. 2017. Binning-refiner: improving genome bins through the combination of different binning programs. Bioinformatics 33:1873-1875. https://doi.org/10.1093/bioinformatics/btx086.

60. Uritskiy GV, DiRuggiero J, Taylor J. 2018. MetaWRAP — a flexible pipeline for genome-resolved metagenomic data analysis. Microbiome 6:158 https://doi.org/10.1186/s40168-018-0541-1.

61. Sieber CMK, Probst AJ, Sharrar A, Thomas BC, Hess M, Tringe SG, Banfield JF. 2018. Recovery of genomes from metagenomes via a dereplication, aggregation and scoring strategy. Nat Microbiol 3:836-843. https://do org/10.1038/s41564-018-0171-1.

62. Olm MR, Brown CT, Brooks B, Banfield JF. 2017. DRep: a tool for fast and accurate genomic comparisons that enables improved genome recovery from metagenomes through de-replication. ISME J 11:2864-2868. https:// doi.org/10.1038/ismej.2017.126.

63. Karst SM, Kirkegaard RH, Albertsen M. 2016. Mmgenome: a toolbox for reproducible genome extraction from metagenomes. bioRxiv https://doi .org/10.1101/059121.

64. Parks DH, Rinke C, Chuvochina M, Chaumeil PA, Woodcroft BJ, Evans PN Hugenholtz P, Tyson GW. 2017. Recovery of nearly 8,000 metagenomeassembled genomes substantially expands the tree of life. Nat Microbiol 2:1533-1542. https://doi.org/10.1038/s41564-017-0012-7.

65. Bosi E, Donati B, Galardini M, Brunetti S, Sagot MF, Lió P, Crescenzi P, Fani R, Fondi M. 2015. MeDuSa: a multi-draft based scaffolder. Bioinformatics 31:2443-2451. https://doi.org/10.1093/bioinformatics/btv171.

66. Parks DH, Imelfort M, Skennerton CT, Hugenholtz P, Tyson GW. 2015 CheckM: assessing the quality of microbial genomes recovered from isolates, single cells, and metagenomes. Genome Res 25:1043-1055. https:// doi.org/10.1101/gr.186072.114

67. Nayfach S, Rodriguez-Mueller B, Garud N, Pollard KS. 2016. An integrated metagenomics pipeline for strain profiling reveals novel patterns of bacterial transmission and biogeography. Genome Res 26:1612-1625. https://do .org/10.1101/gr.201863.115.

68. Chaumeil P-A, Mussig AJ, Hugenholtz P, Parks DH. 2019. GTDB-Tk: a toolkit to classify genomes with the Genome Taxonomy Database. Bioinformatics 36:1925-1927. https://doi.org/10.1093/bioinformatics/btz848.

69. Hyatt D, Chen G-L, Locascio PF, Land ML, Larimer FW, Hauser LJ. 2010. Prodigal: prokaryotic gene recognition and translation initiation site identification. BMC Bioinformatics 11:119. https://doi.org/10.1186/1471-2105-11-119.

70. Altschul SF, Gish W, Miller W, Myers EW, Lipman DJ. 1990. Basic loca alignment search tool. J Mol Biol 215:403-410. https://doi.org/10.1016/ S0022-2836(05)80360-2.

71. Sayers EW, Cavanaugh M, Clark K, Ostell J, Pruitt KD, Karsch-Mizrachi I. 2019. GenBank. Nucleic Acids Res 47:D94-D99. https://doi.org/10.1093/ nar/gky989.

72. UniProt Consortium. 2019. UniProt: a worldwide hub of protein knowledge. Nucleic Acids Res 47:D506-D515. https://doi.org/10.1093/nar/gky1049.
73. Ogata H, Goto S, Sato K, Fujibuchi W, Bono H, Kanehisa M. 1999. KEGG: Kyoto Encyclopedia of Genes and Genomes. Nucleic Acids Res 27:29-34. https://doi.org/10.1093/nar/27.1.29.

74. Punta M, Coggill PC, Eberhardt RY, Mistry J, Tate J, Boursnell C, Pang N, Forslund K, Ceric G, Clements J, Heger A, Holm L, Sonnhammer ELL, Eddy SR, Bateman A, Finn RD. 2012. The Pfam protein families database. Nucleic Acids Res 40:D290-D301. https://doi.org/10.1093/nar/gkr1065.

75. Huerta-Cepas J, Szklarczyk D, Forslund K, Cook H, Heller D, Walter MC, Rattei T, Mende DR, Sunagawa S, Kuhn M, Jensen LJ, von Mering C, Bork P. 2016. eggNOG 4.5: a hierarchical orthology framework with improved functional annotations for eukaryotic, prokaryotic and viral sequences. Nucleic Acids Res 44:D286-D293. https://doi.org/10.1093/nar/gkv1248.

76. Doron S, Melamed S, Ofir G, Leavitt A, Lopatina A, Keren M, Amitai G, Sorek R. 2018. Systematic discovery of antiphage defense systems in the microbial pangenome. Science 359:eaar4120. https://doi.org/10.1126/science.aar4120.

77. Parks DH, Chuvochina M, Waite DW, Rinke C, Skarshewski A, Chaumeil P A, Hugenholtz P. 2018. A standardized bacterial taxonomy based on genome phylogeny substantially revises the tree of life. Nat Biotechnol 36: 996-1004. https://doi.org/10.1038/nbt.4229.

78. Stamatakis A. 2014. RAxML version 8: a tool for phylogenetic analysis and post-analysis of large phylogenies. Bioinformatics 30:1312-1313. https:// doi.org/10.1093/bioinformatics/btu033.

79. Darriba D, Taboada GL, Doallo R, Posada D. 2011. ProtTest 3: fast selection of best-fit models of protein evolution. Bioinformatics 27:1164-1165 https://doi.org/10.1093/bioinformatics/btr088.

80. Letunic I, Bork P. 2016. Interactive tree of life (iTOL) v3: an online tool for the display and annotation of phylogenetic and other trees. Nucleic Acids Res 44:W242-W245. https://doi.org/10.1093/nar/gkw290.

81. Li H, Durbin R. 2010. Fast and accurate long-read alignment with Burrows-Wheeler transform. Bioinformatics 26:589-595. https://doi.org/10 .1093/bioinformatics/btp698.

82. Li H, Handsaker B, Wysoker A, Fennell T, Ruan J, Homer N, Marth G, Abecasis G, Durbin R, 1000 Genome Project Data Processing Subgroup. 2009. The Sequence Alignment/Map format and SAMtools. Bioinformatics 25:2078-2079. https://doi.org/10.1093/bioinformatics/btp352.

83. R Core Team. 2014. R: a language and environment for statistical computing. R Foundation for Statistical Computing, Vienna, Austria.

84. Gloor GB, Macklaim JM, Pawlowsky-Glahn V, Egozcue JJ. 2017. Microbiome datasets are compositional: and this is not optional. Front Microbiol 8:2224. https://doi.org/10.3389/fmicb.2017.02224.

85. Palarea-Albaladejo J, Martín-Fernández JA. 2015. zCompositions - R package for multivariate imputation of left-censored data under a compositional approach. Chemom Intell Lab Syst 143:85-96. https://doi.org/ 10.1016/j.chemolab.2015.02.019.

86. Quinn TP, Richardson MF, Lovell D, Crowley TM. 2017. propr: an R-package for identifying proportionally abundant features using compositional data analysis. Sci Rep 7:16252. https://doi.org/10.1038/s41598-017-16520-0.

87. Wei T, Simko V. 2017. R Package "corrplot": visualization of a correlation matrix. https://cran.r-project.org/web/packages/corrplot/index.html.

88. Gabor C, Tamas N. 2006. The igraph software package for complex network research. InterJournal Complex Syst 1695.

89. Oksanen J, Blanchet FG, Friendly $M$, Kindt $R$, Legendre $P$, Mcglinn $D$ Minchin PR, O'Hara RB, Simpson GL, Solymos P, Henry M, Stevens H, Szoecs E, Maintainer HW. 2019. Package "vegan" community ecology package. https://cran.r-project.org/web/packages/vegan/vegan.pdf.

90. Nakazawa M. 2021. Fmsb: functions for medical statistics book with some demographic Data. R package version 0.7.1. https://cran.r-project.org/ package $=\mathrm{fmsb}$.

91. Beck MW. 2017. vif_fun.r. https://gist.github.com/fawda123/4717702.

92. Hamilton NE, Ferry M. 2018. ggtern: ternary diagrams using ggplot2. J Stat Softw 87:1-17. https://doi.org/10.18637/jss.v087.c03.

93. Bower CE, Holm-Hansen T. 1980. A salicylate-hypochlorite method for determining ammonia in seawater. Can J Fish Aquat Sci 37:794-798. https://doi.org/10.1139/f80-106.

94. Grasshoff K, Ehrhardt M, Kremling K. 1983. Methods of seawater analysis, 2nd ed. Verlag Chemie, Basel, Switzerland.

95. Eaton A, Clesceri L, Greenberg A, Franson M, American Public Health Association, American Water Works Association, Water Environment Federation. 1998. Standard methods for the examination of water and wastewater, 20th ed. American Public Health Association, Washington, DC. 